\section{International Scientific Journal Theoretical \& Applied Science}

p-ISSN: 2308-4944 (print) e-ISSN: 2409-0085 (online)

Year: $2014 \quad$ Issue: $10 \quad$ Volume: 18

Published: $30.10 .2014 \quad$ http://www.T-Science.org
Anatoly Aleksandrovich Naumov Docent, Candidate of Technical Sciences, Center of Applied Mathematical Research,

Novosibirsk, Russia,

a_a_naumov@mail.ru

Alexey Anatolievich Naumov General Director, Vector, Surgut, Russia, alexey.naumov@gmail.com

SECTION 31. Economic research, finance, innovation.

\title{
THE CONTEXT DEPENDENCE OF INVESTMENT PROJECTS AND OPTIMIZATION OF THEIR PARAMETERS
}

Abstract: The paper discusses the results of a study of methods of optimizing the parameters of the investment projects. Used parameterization of projects on the basis of detailed financial flows.

Key words: Investment projects, financial flows, the method detailed flows, efficiency, optimization projects, modeling.

Language: Russian

Citation: Naumov AA, Naumov AA (2014) THE CONTEXT DEPENDENCE OF INVESTMENT PROJECTS AND OPTIMIZATION OF THEIR PARAMETERS. ISJ Theoretical \& Applied Science 10 (18): 56-58. doi: http://dx.doi.org/10.15863/TAS.2014.10.18.14

УДК 336.77: 330.42

\section{КОНТЕКСТНАЯ ЗАВИСИМОСТЬ ИНВЕСТИЦИОННЫХ ПРОЕКТОВ И ОПТИМИЗАЦИЯ ИХ ПАРАМЕТРОВ}

Аннотация: В работе рассмотрены результаты исследования методов оптимизации параметров инвестиционных проектов. Использована параметризачия проектов на основе метода детализации финансовых потоков.

Ключевые слова: Инвестиционные проекты, финансовые потоки, метод детализации потоков, эффективность, оптимизация проектов, моделирование.

Постановка задачи.

Пусть для некоторого проекта известны его входной и выходной финансовые потоки в виде: $F_{\text {in }}(t), t=t_{0}, t_{1}, t_{2}, \ldots,\left(t_{m}=T\right),-$ входной поток, $F_{\text {out }}(t), t=t_{0}, t_{1}, t_{2}, \ldots,\left(t_{m}=T\right), \quad-\quad$ выходной поток. Задача состоит в оценивании эффективности этого проекта и в нахождении таких его параметров (объемов собственных и заемных средств, ставок заимствования и внешнего использования и пр.), которые доставляли бы максимальное значение критерию эффективности проекта. Такая задача обобщает известные задачи оптимизации структуры капитала, оптимизации параметров финансового рычага и др.

Параметризация проекта и метод детализации его потоков.

Воспользуемся методом детализации потоков и оценим на его основе эффективность проекта (см. подробнее в [1-3]). Так, при детализации выходного потока проекта будут получены два новых потока $\left(F_{\text {out,IP }}^{\Sigma}\right.$ и $F_{\text {out,EP }}^{\Sigma}-$ внутреннего и внешнего использования финансовых средств проекта) и два соответствующих им вектора параметров $-\pi_{\text {out }, I P}^{\Sigma}$ и $\pi_{o u t, E P}^{\Sigma}$. Эти параметры представляют собой ставки, по которым элементы $F_{o u t, I P}^{\Sigma}$ ожидают их использования в качестве погашений заимствований $F_{i n, D F}^{\Sigma}$ (это параметры $\pi_{o u t, I P}^{\Sigma}$ ), а элементы $F_{o u t, E P}^{\Sigma}-$ используются во внешних проектах (за это отвечают параметры $\pi_{o u t, E P}^{\Sigma}$ ). Аналогичные векторы параметров сопровождают и компоненты вектора $F_{i n, D F}^{\Sigma}$ (и векторов $\left.F_{i n, D F, i}, i=0,1,2, \ldots, m\right)$, которые получаются при детализации входного потока проекта. Для этих векторов параметры характеризуют элементы входного потока: ставки заимствования $r_{i n, i}, i=$ $0,1,2, \ldots, m$, количество временных тактов погашения кредитов $n_{i}, i=0,1,2, \ldots, m$ и т.д. 
Уточним вид векторов параметров для каждого из потоков проекта: 1) для элементов $\mathrm{F}_{\text {in }}(\mathrm{t}), \mathrm{t}=\mathrm{t}_{0}, \mathrm{t}_{1}, \mathrm{t}_{2}, \ldots, \mathrm{t}_{\mathrm{m}}$, параметры имеют вид $\pi_{\mathrm{in}, \mathrm{DF}, \mathrm{i}}=\left(\mathrm{t}_{\mathrm{i}} ; \mathrm{T}_{\mathrm{i}} ; \mathrm{n}_{\mathrm{i}} ; \mathrm{r}_{\mathrm{in}, \mathrm{i}}\right), \mathrm{i}=0,1,2, \ldots, \mathrm{m}$, причем, выполняется равенство $\mathrm{n}_{\mathrm{i}}=\mathrm{T}_{\mathrm{i}}-\mathrm{t}_{\mathrm{i}}, \quad \mathrm{i}=$ $0,1,2, \ldots, \mathrm{m} ; \quad r_{i n}-$ ставка заимствования инвестируемых в проект финансовых средств; множество допустимых значений для этих параметров обозначим через $\prod_{\mathrm{in,DF}, \mathrm{i}}, \mathrm{i}=$ $0,1,2, \ldots, \mathrm{m}$; для элементов потока, которые не являются заемными средствами параметры равны $\pi_{\mathrm{in}, \mathrm{DF}, \mathrm{i}}=\left(\mathrm{t}_{\mathrm{i}} ; \mathrm{T}_{\mathrm{i}}=\mathrm{t}_{\mathrm{i}} ; \mathrm{n}_{\mathrm{i}}=0 ; \mathrm{r}_{\mathrm{in}, \mathrm{i}}=0\right), \quad \mathrm{i}=$ $0,1,2, \ldots, \mathrm{m} ; 2$ ) для потока $\mathrm{F}_{\mathrm{in}, \mathrm{DF}}^{\Sigma}=\oplus_{\mathrm{i}=0}^{\mathrm{m}} \mathrm{F}_{\mathrm{in}, \mathrm{DF}, \mathrm{i}}$ вектор параметров будет иметь вид: $\pi_{\mathrm{in,DF}}^{\Sigma}=$ $\left(\pi_{\mathrm{in}, \mathrm{DF}, 0}, \pi_{\mathrm{in,DF}, 1}, \ldots, \pi_{\mathrm{in}, \mathrm{DF}, \mathrm{m}}\right) ; \quad$ заметим, что множество допустимых значений для этого вектора параметров имеет вид: $\prod_{\mathrm{in,DF}}^{\Sigma}=$ $\otimes_{\mathrm{i}=0}^{\mathrm{m}} \prod_{\mathrm{in,DF}, \mathrm{i}} ; \quad$ здесь «®» - символ прямого произведения множеств; 3) для элементов выходного потока $\mathrm{F}_{\text {out }}(\mathrm{t}), \mathrm{t}=\mathrm{t}_{0}, \mathrm{t}_{1}, \mathrm{t}_{2}, \ldots, \mathrm{t}_{\mathrm{m}}$, параметры будут характеризовать условия использования доходов: $\pi_{\text {out,IP }}^{\Sigma}$ - параметры для данного проекта, $\pi_{\text {out,EP }}^{\Sigma}-$ параметры для использования финансовых средств в других проектах; например, вид параметров может быть таким: $\quad \pi_{\text {out }, I P, i}=\left(t_{i} ; T_{i} ; n_{i} ; r_{\text {out }, \mathrm{i}} ; r_{\text {del, }}\right), \quad \mathrm{i}=$ $0,1,2, \ldots, \mathrm{m}$; здесь $t_{i}-$ время начала использования дохода в качестве элементов входного потока; $\mathrm{T}_{\mathrm{i}}-$ время окончания их использования; $\mathrm{n}_{\mathrm{i}}-$ длительности использования во временных тактах; $r_{\text {out,i }}$ - ставка, по которой средства передаются в качестве инвестиционных средств; $\mathrm{r}_{\mathrm{del}, \mathrm{i}}$ - ставка, по которой средства ожидают вложений в проект; тогда $\pi_{\text {out,IP }}^{\Sigma}=$ $\left(\pi_{\text {out }, \text { IP }, 0}, \pi_{\text {out }, \text { IP }, 1}, \ldots, \pi_{\text {out }, \text { IP, }, \mathrm{m}}\right) \quad$ и $\quad \prod_{\text {out,IP }}^{\Sigma}=$ $\otimes_{\mathrm{i}=0}^{\mathrm{m}} \prod_{\text {out,IP,i }} ; \quad$ аналогично для элементов выходного потока, выводимых из данного проекта в другие проекты: $\pi_{\text {out,EP,i }}=\left(\mathrm{t}_{\mathrm{i}} ; \mathrm{T}_{\mathrm{i}} ; \mathrm{n}_{\mathrm{i}} ; \mathrm{r}_{\mathrm{out}, \mathrm{i}} ; \mathrm{r}_{\mathrm{del}, \mathrm{i}}\right)$, $\mathrm{i}=0,1,2, \ldots, \mathrm{m} ; \quad \pi_{\text {out,EP }}^{\Sigma}=$ $\left(\pi_{\text {out }, \mathrm{EP}, 0}, \pi_{\text {out }, \mathrm{EP}, 1}, \ldots, \pi_{\text {out }, \mathrm{EP}, \mathrm{m}}\right) \quad$ и $\quad \prod_{\text {out,EP }}^{\Sigma}=$ $\otimes_{\mathrm{i}=0}^{\mathrm{m}} \prod_{\text {out,EP,i }}$; заметим, что в последнем случае $\mathrm{r}_{\text {out,i }}, \quad \mathrm{i}=0,1,2, \ldots, \mathrm{m}$, - доходности внешних относительно данного проекта проектов. Именно в связи с тем обстоятельством, что во множество параметров проекта входят параметры смежных проектов, имеет смысл рассматривать контекстную зависимость. Заметим, что классические методы анализа проектов на эффективность такую зависимость не учитывают. Конечно, это один из их недостатков [3].

\section{Оптимизация проекта.}

Сформулируем задачу оптимизации параметров проекта. Обозначим множество критериев задачи в виде вектора $\vec{Q}=$ $\left(\mathrm{Q}_{1}, \mathrm{Q}_{2}, \ldots, \mathrm{Q}_{\mathrm{k}}\right)$, где $\mathrm{Q}_{\mathrm{i}}, \mathrm{i}=0,1,2, \ldots, \mathrm{k}$, - критерии эффективности проекта (например, $N F V$, NFVDF, $I R R, P P$ и др.). Тогда оптимизацию параметров финансовых потоков проекта можно осуществить в соответствии с задачей на экстремум:

$\vec{Q}=\left(Q_{1}, Q_{2}, \ldots, Q_{k}\right) \underset{\pi_{\text {in,DF }}^{\Sigma}, \pi_{\text {out }, I P}^{\Sigma}, \pi_{\text {out }, \mathrm{EP}}^{\Sigma}}{\longrightarrow}$ extremum, при ограничениях: $\pi_{\mathrm{in,DF}}^{\Sigma} \in \prod_{\mathrm{in,DF}}^{\Sigma} ; \quad \pi_{\text {out,IP }}^{\Sigma} \in$ $\prod_{\text {out }, \text { IP }}^{\Sigma} ; \pi_{\text {out,EP }}^{\Sigma} \in \prod_{\text {out,EP. }}^{\Sigma}$.

Отметим некоторые особенности этой оптимизационной задачи и ее переменных. Задача представляет собой задачу дискретного программирования (более точно - выбора лучшего варианта, перебора вариантов) с векторным критерием. Вектор параметров $\pi_{\text {out,IP }}^{\Sigma}$, а следовательно, и зависящий от него вектор $\pi_{\text {out,ЕР }}^{\Sigma}$ формируются на основании детализированного входного потока и его параметров $-\pi_{\mathrm{in,DF}}^{\Sigma}$. Поэтому сначала необходимо сформировать множество параметров $\prod_{\mathrm{in,DF}}^{\Sigma}$, a уже на его основе - множества $\prod_{\text {out,IP }}^{\Sigma}$ и $\prod_{\text {out,EP }}^{\Sigma}$ В некоторые моменты времени векторы параметров $\quad \pi_{\mathrm{in,DF}, \mathrm{i}}=\left(\mathrm{t}_{\mathrm{i}} ; \mathrm{T}_{\mathrm{i}} ; \mathrm{n}_{\mathrm{i}} ; \mathrm{r}_{\mathrm{in}, \mathrm{i}}\right), \quad \mathrm{i}=$ $0,1,2, \ldots, \mathrm{m}$, или векторы в свертках $\pi_{\text {out,IP }}^{\Sigma}$ или $\pi_{\text {out,EP }}^{\Sigma}$ могут отсутствовать, поскольку в соответствующие моменты времени имеют нулевые значения элементы входных или выходных потоков. При необходимости в качестве элементов векторов параметров могут быть включены и другие характеристики потоков: значения входных потоков $\mathrm{F}_{\text {in }}(\mathrm{t}), \mathrm{t}=$ $\mathrm{t}_{0}, \mathrm{t}_{1}, \mathrm{t}_{2}, \ldots, \mathrm{t}_{\mathrm{m}}$, времен к которым они относятся, если они отличаются от времен начала этапов выплат по кредитам, значения выходных потоков $\mathrm{F}_{\text {out }}(\mathrm{t}), \mathrm{t}=\mathrm{t}_{0}, \mathrm{t}_{1}, \mathrm{t}_{2}, \ldots, \mathrm{t}_{\mathrm{m}}$, и т.д. Кроме этого, одним и тем же элементам входных или выходных потоков для фиксированных значений $\mathrm{t} \in\left\{\mathrm{t}_{0}, \mathrm{t}_{1}, \mathrm{t}_{2}, \ldots, \mathrm{t}_{\mathrm{m}}\right\}, \quad$ может соответствовать несколько наборов векторов параметров; это может быть объяснено, например, тем обстоятельством, что заемные средства поступают из нескольких источников финансирования, а доходы распределяются по нескольким альтернативным внешним для данного проекта проектам.

\section{References:}


1. Naumov AA (2013) Optimizatsiya strukturyi zaimstvovaniy i vlozheniy dohodov investitsionnogo proekta. Materials of the ISPC «Advances in techniques\&technologies», 30.10.2013, Milan, Italy. ISJ Theoretical \& Applied Science 10(6): 133-136. doi: http://dx.doi.org/10.15863/TAS.2013.10.6.20

2. Naumov AA (2013) Ispolzovanie metoda detalizatsii finansovyih potokov $\mathrm{v}$ zadachah otsenivaniya effektivnosti proektov. Finansovaya analitika: problemyi i resheniya. No. 48 (186), pp. 35-41.

3. Naumov AA (2013) Metodyi analiza i sinteza investitsionnyih proektov. Effektivnost, riski, upravlenie. LAP LAMBERT Academic Publishing. pp. 356.

4. Naumov AA (2014) Otsenivanie effektivnosti integrirovannyih proektov. Finansovaya analitika: problemyi i resheniya. No. 8 (194), pp. 36-43.

5. Naumov AA (2014) Analiz kriteriev effektivnosti investitsionnyih proektov. Materials of the ISPC «Modern mathematics in science», 30.06.2014, Caracas, Venezuela. ISJ Theoretical \& Applied Science 6 (14): 92-94. doi:

http://dx.doi.org/10.15863/TAS.2014.06.14.19

6. Naumov AA (2014) Razmyityie proektyi: modeli $i$ algoritmyi. Materials of the ISPC «The development of electronics \& mechanics», 30.05.2014, Barcelona, Venezuela. ISJ
Theoretical\&Applied Science 5 (13), pp. 97-99. doi:

http://dx.doi.org/10.15863/TAS.2014.05.13.16

7. Naumov AA (2014) Metodyi adaptatsii biznesprotsessov. Materials of the ISPC «The development of electronics \& mechanics», 30.05.2014, Barcelona, Venezuela. ISJ Theoretical\&Applied Science 5 (13): 100-102. doi:

http://dx.doi.org/10.15863/TAS.2014.05.13.17

8. Naumov AA (2013) Matematicheskie modeli sistemyi upravleniya prodvizheniem ITproduktov banka. Materials of the International Scientific Practical Conference «Theoretical Research», 30.07.2013, Stuttgart, Germany. ISJ Theoretical \& Applied Science 7(3): 95-102. doi:

http://dx.doi.org/10.15863/TAS.2013.07.3.13

9. Naumov AA (2013) Pereraspredelenie obschego dohoda mezhdu uchastnikami integrirovannogo proekta. Materials of the ISPC «Advances in techniques\&technologies», 30.10.2013, Milan, Italy. ISJ Theoretical \& Applied Science 10(6): 129-132. doi: http://dx.doi.org/10.15863/TAS.2013.10.6.19

10. Spisok trudov (2014) [Elektronnyiy resurs]. URL:

https://sites.google.com/site/anatolynaumov201 1/home/spisok-trudov-list-of-papers (data obrascheniya: 27.10.2014). 